\title{
When the Price Is Your Privacy A Security Analysis of Two Cheap loT Devices
}

Favaretto, Margherita; Tran Anh, Tu; Kavaja, Juxhino; De Donno, Michele; Dragoni, Nicola

\section{Published in:}

Proceedings of $6<$ sup $>$ th $</$ sup $>$ International Conference in Software Engineering for Defence Applications SEDA 2018

Link to article, DOI:

10.1007/978-3-030-14687-0_6

Publication date:

2020

Document Version

Peer reviewed version

Link back to DTU Orbit

\section{Citation (APA):}

Favaretto, M., Tran Anh, T., Kavaja, J., De Donno, M., \& Dragoni, N. (2020). When the Price Is Your Privacy: A Security Analysis of Two + Gheap loT Devices. In M. Mazzara, A. Messina, A. Sillitti, G. Succi, \& P. Ciancarini (Eds.), Proceedings of 6 International Conference in Software Engineering for Defence Applications - SEDA 2018 (pp. 55-75). Springer. Advances in Intelligent Systems and Computing Vol. 925 https://doi.org/10.1007/978-3-030-14687-0 6

\section{General rights}

Copyright and moral rights for the publications made accessible in the public portal are retained by the authors and/or other copyright owners and it is a condition of accessing publications that users recognise and abide by the legal requirements associated with these rights.

- Users may download and print one copy of any publication from the public portal for the purpose of private study or research.

- You may not further distribute the material or use it for any profit-making activity or commercial gain

- You may freely distribute the URL identifying the publication in the public portal 


\title{
When the Price is Your Privacy: A Security Analysis of Two Cheap IoT Devices
}

\author{
Margherita Favaretto ${ }^{1}$, Tu Tran Anh ${ }^{1}$, Juxhino Kavaja ${ }^{1}$, Michele De Donno ${ }^{1}$, \\ and Nicola Dragoni ${ }^{12}$ \\ 1 DTU Compute, Technical University of Denmark, Denmark \\ s170065@student.dtu.dk, s156015@student.dtu.dk, juxk@dtu.dk, mido@dtu.dk, \\ ndra@dtu.dk \\ 2 Centre for Applied Autonomous Sensor Systems (AASS), \\ Örebro University, Sweden
}

\begin{abstract}
The Internet of Things (IoT) is shaping a world where devices are increasingly interconnected, cheaper, and ubiquitous. The more we move toward this world, the more cybersecurity becomes paramount. Nevertheless, we argue that there exists a category of IoT devices which commonly overlooks security, despite dealing with sensitive information. In order to demonstrate this, in this work, we present the results of the security assessments we performed on two IoT devices that we consider emblematic of such category: the Rohs K88h smartwatch and the Sricam SP009 IP camera. The results demonstrate the existence of critical vulnerabilities that could be easily exploited, even by non-expert attackers, for extracting sensitive information and severely impacting on user's privacy.
\end{abstract}

\section{Introduction}

The Internet of Things (IoT) is increasingly branching out in our daily lives. It is changing our cities [1, homes [2, 3], industries [4, 5, transports [6], and healthcare 7, 8, just to name a few. This trend is not going to stop, as it is estimated that by 2020 the number of IoT devices is expected to reach 21 billion 9 .

This relentless growth of IoT demonstrates that the advantages resulting from such paradigm are well known. Nevertheless, since the IoT is far from being perfect, it is paramount to cope with such enthusiasm and increase the awareness about the issues associated with its usage. Among these issues, one of the most critical is cybersecurity.

The security concerns associated with the IoT have been extensively discussed in the literature 10 12. However, it is our strong belief that there still exists a lack of information about the importance of appropriately securing IoT devices. This is especially the case for cheap IoT devices, where both users and manufacturers do not realize that in most cases, even if such devices have a small economic value, the quantity of sensitive information they store turns them into invaluable devices that need to be protected at any cost. 


\subsection{Contribution}

The main motivation behind this work is to raise awareness in relation to the severe privacy issues arising when IoT devices are not properly secured. We argue that there exists a category of IoT devices that can be easily exploited by attackers since lacking adequate security mechanisms. This category is composed of those IoT devices which are used by consumers in their daily lives and that are perceived as accessories, and not as primary goods. We claim that there are two properties that make such devices particularly important from a security perspective and at the same time vulnerable:

- Ubiquitousness: although such devices are considered futile, since not vital for consumers, from a security perspective they can not be considered as such. Indeed, by accompanying users in every step of their daily lives, they can acquire large amounts of sensitive information which turns them into critical devices that need to be protected.

- Cheapness: the only criteria employed by consumers for choosing which device to buy, is essentially based on the highest number of functionalities they can get at the lowest price. This implies that producers strive to reduce costs as much as possible, which usually implies sacrificing security.

On the same line of some previous work 13 14, in order to demonstrate the great potential of being attacked that this category of IoT devices has, in this paper we report the results of the security analysis we performed on two different IoT devices: the Rohs K88h smartwatch, the Sricam SP009 IP camera.

Both devices have the property of being among the cheapest in their market segment and of being ubiquitous (i.e. supporting and accompanying users in their daily activities). We emphasize that these were the only criteria we adopted in choosing such devices for performing our security assessments.

\subsection{Paper structure}

The rest of this paper is organized as follows. Section 2 presents the security assessment that has been performed on the Rohs K88h smartwatch. Section 3 describes the vulnerabilities we identified on the Sricam SP009 IP camera. Internally, these sections, are both structured in a similar way: initially, the most relevant works dealing with the privacy issues of such devices are briefly presented; then, an overview of the devices and of the tools used for performing their security assessments is given; finally, the identified vulnerabilities are presented together with possible solutions. Section 4 concludes the paper and outlines the topics on which we are currently working for extending this work.

\section{Security of cheap and ubiquitous IoT devices: a smartwatch case study}

Smartwatches are rapidly becoming widely adopted 15 . These devices are increasingly being used for disparate purposes: remote health monitoring [16, 17, 
activity recognition [18 20, biometric authentication [21, 22, supporting outdoor activities 23] and many others. All these applications are enabled by few characteristics that these devices have: the high number of sensors and functionalities they offer, their proximity to end-users and, most importantly, their reduced cost. Nevertheless, if from the one hand these characteristics allow the widespread adoption of smartwatches, on the other hand, the same properties make such devices insecure. Indeed, the fact that they are equipped with many sensors which are constantly located in the proximity of end-users allows such devices to acquire a lot of sensitive data that need to be protected. However, the need to protect such data collides with the need for reducing production costs. In order to demonstrate this, we performed the security assessment of a device that we consider emblematic of all other devices satisfying the aforementioned characteristics.

The goal of this section is to describe the results of the security assessment that we performed on the Rohs K88h smartwatch together with the vulnerability we identified. Before doing this, in the following subsection, we present the main literature works that dealt with the security issues of smartwatches.

\subsection{Related Works}

In this section, we report some of the literature works discussing the security issues of smartwatches. We mostly focus on manuscripts that take into account how such devices can be exploited for affecting the privacy of users.

Several works in the literature, such as 24 28], demonstrate the possibility to exploit smartwatches sensors for inferring inputs typed on external keyboards. These works show that sensitive information (such as PINs) can be inferred through data sensed from smartwatches. These attacks are considered side-channels attacks and are commonly referred to as keystroke inference attacks.

Differently from the aforementioned works, $\mathrm{Lu}$ et al. 29 show that it is possible to use sensors embedded on smartwatches, not only for inferring inputs typed on external keyboards, but even for discovering PINs typed on the same smartwatch where the sensors are installed.

In 30, Do et al. describe the types of sensitive data that are stored in smartwatches. They also present an attack for extracting these data from smartwatches based on the Android Wear platform. For this category of devices, the authors have been able to exfiltrate data by exploiting a security flaw of the devices bootloader.

Liu and Sun 31 present a survey of attack methodologies that can affect wearable devices. They classify attacks in relation to the security property they affect, focusing on three properties: integrity, authenticity, and privacy.

Analyzing the security of smartwatches is a complex task due to the different existing models, vendors, and functionalities. For this reason, Siboni et al. 32 propose a testbed framework for testing the security properties of wearable devices. The goal of such framework is to test the wearable IoT devices against a given set of security requirements. As we are going to show in the next sections, 
most of the security flaws identified in the devices we considered in our security assessment are basic and common errors that could have been easily discovered by such framework.

Lee et al. 33 perform experimental studies for evaluating the amount of data transferred from host devices (e.g. smartphones) to wearable devices (e.g. smartwatches) after they have been successfully paired with each other. Based on the results of this analysis, a large amount of sensitive information is transferred from host devices to the wearable ones. On top of that, it resulted that such transfers of sensible data are not sufficiently notified to users. Such a situation rises severe privacy concerns as it impedes users from clearly knowing where their data are stored.

In [34, ten different smartwatches are studied from a security perspective. As expected, the results of such study demonstrate a lack of proper security mechanisms and the existence of naive vulnerabilities (such as, insufficient authentication and authorization or lack of mechanisms to protect network services) that can easily be exploited also by non-expert attackers.

In the rest of this section, we are going to describe the results of the security assessment we performed on the Rohs K88h smartwatch. To this aim, in the next subsection, we describe the common scenario in which every smartwatch operates. Hence, we explain how these devices communicate with smartphones and their role in preserving users' privacy. Therefore, we recall some basic concepts of the Bluetooth technology that are going to be fundamental for describing the security vulnerability of the Rohs K88h smartwatch.

\subsection{The typical setting in which smartwatches operate}

Smartwatches are typically connected to a smartphone by means of a Bluetooth connection. Such a connection is used by the smartwatch to receive user's data from the other device (e.g. messages, contacts, notifications and others). However, it is also employed for sending data in the opposite direction. Indeed, by means of their sensors, smartwatches collect several types of data (e.g. sleeping habits, physical activity records and other personal information) that need to be shared with a smartphone for being elaborated and allowing the extraction of useful information. In many cases, the smartphone may also use an Internet connection for outsourcing such data and performing remote elaboration on the Cloud. Fig 1 depicts the relevant entities and communications that have to be considered when working with smartwatches.

In the following, we are only going to consider the Bluetooth communication between the smartwatch and the smartphone. In relation to this, we are going to describe the vulnerability we identified in the smartwatch implementation of the Bluetooth technology.

Bluetooth background In this subsection, we present some basic concepts of the Bluetooth technology that are going to be used in the subsequent sections. For a thorough treatment of Bluetooth and its security implications, we suggest the reader refers to the NIST guideline on Bluetooth security [35]. 


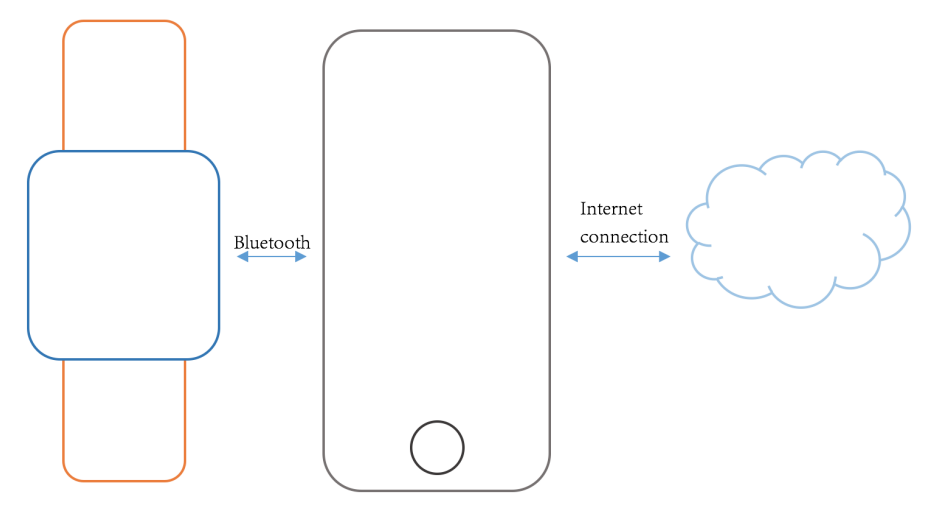

Fig. 1. Relevant entities and communications to consider when dealing with common smartwatches.

Bluetooth is an open standard for short-range radio frequency communication mainly used for establishing wireless personal area networks (WPANs) 35. It is a low-cost and low-power technology operating in the frequency range between 2.4000 gigahertz $(\mathrm{GHz})$ and 2.4835 gigahertz $(\mathrm{GHz})$ [35.

Discoverable and connectable modes are defined by the Bluetooth standard for allowing devices to communicate and connect with each other. In simple, when a device operates in both modes, it replies to inquiries from other devices in such a way to be detectable and to permit the establishment of connections [35]. In a Bluetooth network, it is always possible to distinguish a master device from all other devices which are typically referred to as slave nodes. A master device controls and establishes the network and defines the network frequency hopping scheme 35. The network frequency hopping scheme is a transmission technique that can be seen as a first minimal security mechanism that avoids inference and makes it a bit more difficult (if compared to fixed-frequency transmissions such as IEEE $802.11 \mathrm{~g} / \mathrm{n}$ ) to capture transmissions [35].

From a security perspective, the Bluetooth standard defines only three security services: Authentication, Confidentiality and Authorization. Each Bluetooth device has to select and operate in one Security Mode. There exists 4 Security Modes which defines when the aforementioned security services (authentication, confidentiality and authorization) are initiated [35. We briefly summarize the properties of each of them [35]:

1. Security Mode 1: security functionalities are never started. By employing such Security Mode, Bluetooth devices cannot be distinguished, moreover, no mechanisms for preventing the establishment of connections can be applied.

2. Security Mode 2: in this case, security functionalities can be initiated after link establishment but before logical channel establishment. This is a service level-enforced security mode where authentication, encryption and authorization are used. 
3. Security Mode 3: in this mode, security functionalities have to be started before the physical link is established.

4. Security Mode 4: this is another case of service-level enforced security mode where security functionalities are initiated after physical and logical link setup.

In order to initiate security services (especially authentication and confidentiality), a secret symmetric key, also known as link key, has to be generated. The process of generation of such key is known as pairing process. For the sake of completeness it should be mentioned that Security Modes 2 and 3 perform the pairing process by means of a method called Personal Identification Number (PIN) Pairing (also known as Legacy Pairing); while Security Mode 4 uses the Secure Simple Pairing (SSP) which is meant to simplify the pairing process 35 .

We are not going to detail more about the security of the Bluetooth technology since, as we are going to describe in the next sections, the vulnerability of the smartwatch we analyzed is related to the use of the basic and totally insecure Secure Mode 1. For this reason, given the focus of this paper, it makes no sense to dive into more advanced topics when there still exists devices not considering security at all. We strongly believe that this total lack of security awareness is common in these categories of IoT devices. For this reason, we find paramount to document these issues and contribute to make aware of how much security is neglected together with the devastating impact such problems can have on privacy.

Although the issue we identified for the Rohs K88h smartwatch is not strictly related to any vulnerability of the Bluetooth technology itself (but rather to the lack of knowledge in using it), we underline that there exist several others sophisticated attacks and vulnerabilities. An introduction to these issues is presented in the NIST guide to Bluetooth Security [35].

\subsection{Rohs K88h Smartwatch overview}

The smartwatch we used in performing our security assessment is the Rohs K88h smartwatch. At the time in which these experiments have been performed (October 2017), this is one of the top 10 cheap smartwatch alternatives in 2017 [36, it is placed 62th in the list of Amazon's bestseller "heart rate monitors", 127th in the category of "Fitness technology", and number 1414th in the "smartwatches" category [37.

The Rohs K88h smartwatch uses a MTK2502C processor which is widely adopted by different smartwatch manufacturers. It runs the Nucleus Operating System $(\mathrm{OS})^{3}$, which is commonly used as alternative to Wear $\mathrm{OS}^{4}$ or watchOS ${ }^{5}$. It is equipped with a touch screen, microphone, speaker, Bluetooth 4.0 adapter, and accelerometer. Among the most interesting functionalities it offers we find:

\footnotetext{
3 https://www.mentor.com/embedded-software/nucleus/

4 https://wearos.google.com

5 https://www.apple.com/lae/watchos/
} 
heart rate monitoring, tracking of burned calories, pedometer, sleep monitoring and remote controlling. In Tab. 1 a summary of the hardware specifications of this smartwatch are reported, while in Tab. 2 the main functionalities it offers are summarized.

We chose to evaluate this smartwatch for two main reasons: it is one of the cheapest devices in its market segment and offers numerous features. In other words, we considered it as emblematic of all those devices having the two aforementioned proprieties. It is our strong belief that these characteristics coexist in detriment of security. In order to demonstrate this, we decided to select an IoT device satisfying such criteria and performing a security assessment for verifying what is the real cost associated with the use of cheap and ubiquitous IoT devices. As expected, during our assessment, we found a severe security issue that is not rooted or caused by the used technologies itself, but, instead, caused by misinformation and inattention in implementing such technology in building the smartwatch. Hence, we argue that the potential impact of such naive issues is huge: they can easily be exploited also by non-expert attackers for acquiring a large amount of sensible information.

In the next section we describe the analysis we performed on the Bluetooth connection enabled by the smartwatch and present the vulnerability we identified at this level together with how it could be exploited for affecting the users' privacy.

Table 1. Rohs K88h Hardware specifications.

\begin{tabular}{ll}
\hline \hline CPU & MTK2502C \\
Screen & IPS Capacitive touch screen \\
Battery & 300mAh Li-battery \\
Bluetooth & BT4.0LE \\
Speaker & 1511 ACC Speaker \\
Microphone & YES \\
Memory & $128 \mathrm{MB}+64 \mathrm{MB}$ \\
Accelerometer & YES \\
\hline
\end{tabular}

\subsection{The vulnerability}

The security assessment we performed on the Rohs K88h smartwatch showed a lack of protection of the Bluetooth communication. In this section, we present the tools, commands and steps we followed for identifying this issue.

In order to analyze the Bluetooth connection of the smartwatch, we used common commands available on Kali Linux 2017.2. By using the hcitool scan command, we were able to locate the smartwatch Bluetooth device address 
Table 2. List of Rohs K88h offered features.

\begin{tabular}{|c|c|}
\hline Features & Description \\
\hline \multicolumn{2}{|c|}{ Heart rate monitor Measurement of heart reat } \\
\hline Pedometer & Real time step count \\
\hline Calories tracking & Counting of burned calories \\
\hline Synchronization & $\begin{array}{l}\text { Possibility to pair the smartwatch } \\
\text { with iOS \& Android based smart- } \\
\text { phones }\end{array}$ \\
\hline Remote control & $\begin{array}{l}\text { Access to personal assistants (such } \\
\text { as Siri and Cortana) }\end{array}$ \\
\hline Sleep monitor & Track sleep quality \\
\hline Other & $\begin{array}{l}\text { Calculator, Alarm, Remote camera, } \\
\text { Remote music, Stopwatch, IP54, } \\
\text { Call log, Dial, Phonebook etc. }\end{array}$ \\
\hline
\end{tabular}

(BD_ADDR) and corresponding alias even if the device was not set in discoverable mode. Then, we used the sdptool browse command to inspect the services offered by the device. At this point, we tried to establish a radio frequency communication (RFCOMM) 38 connection in order to verify whether a pairing process would start and notify the end-users of an attempt to connect with the smartwatch. For this purpose, we used the rfcomm connect command without the specification of any channel on which to establish the connection. In this way, not only we were able to establish a connection with the smartwatch, but we also did it in a completely silent way, i.e. no messages were generated from the smartwatch to, at least, notify the establishment of a new connection to the end-user. Since no action was required by the end-user (neither to accept the connection nor to enter a PIN code), we infer that no paring process occurs, which implies that the lowest level of security (Security Mode 1) is used by the Bluetooth connection used by the smartwatch 35 .

Application reverse engineering In order to take advantage of this vulnerability and extract sensible information from the smartwatch, we decided to perform the reverse engineering of the client application running on the smartphone that enables the exchange of data between the smartwatch and smartphone. Our goal is to use the reverse engineering process for building a new client application that can allow to silently exfiltrate sensible data from the device. Therefore, in this subsection, we detail how we performed the reverse engineering and the code we were able to recover.

The application that allows the smartphone to interact and control the smartwatch is called Fundo Wear. By means of this application, end-users can create profiles which permit to synchronize the information acquired by the IoT de- 
vice with the smartphone. Examples of such information are: heart rate, burned calories or step counts measurements.

We performed the reverse engineering of the Andorid version of Fundo Wear v. 1.6.0 using Apktoo] Even if part of the source code resulting from the reverse engineering process is obfuscated, we were able to identify the Java classes that allow to establish a connection with the device and read the heart rate measurements. These classes can allow us to replicate the application so that it can be employed as an exploitation tool against the smartwatch.

Although these classes are a core part required for the implementation of the exploitation tool, we have not developed the complete exploitation tool yet. In relation to this, we emphasize that, even if the identified vulnerability can be exploited in several ways (such as by exploiting the services we identified by means of the command sdptool browse), we are currently working on building a complete exploitation tool that takes advantage of the reverse engineering process.

In the next subsection, we present how the vulnerability we identified could be solved. Moreover, we describe simple precautions that can render Bluetooth communications more secure.

\subsection{Possible Solution}

In the previous subsection, we presented the vulnerability we identified in the implementation of the Bluetooth technology used by the Rohs K88h smartwatch. We identified the existence of an extremely dangerous security flaw which allows external users to easily connect to the smartwatch. We believe that this vulnerability could be easily solved by setting the Security Mode of the device at any level higher than the one that is currently used. Nevertheless, we underline that, even by using higher-level Security Mode, there are still possible attacks that can be performed against a secure Bluetooth connection. In relation to this, a list of Bluetooth-specific attacks can be found in 35].

In the rest of this section, we present some basic precautions that can reduce the risks associated with attacks enabled by the Bluetooth connections of IoT devices. We divide them into two categories: the first considers what can be done from the consumer side, while the other presents what manufacturers can do for enhancing the Bluetooth connection security of IoT devices they produce.

\section{Consumers' side precautions}

- change default manufacturing settings (such as default authentication pin code);

- disable discovery of device if not required;

- turn off Bluetooth interface if not used;

- do not accept pairing requests, unless they are trusted;

- install firmware updates.

\footnotetext{
${ }^{6}$ https://ibotpeaches.github.io/Apktool/
} 


\section{Manufacturers' side precautions}

- do not implement Security Mode 1;

- notify users of every new connections that is attempted;

- encrypt data that are transmitted.

This completes the description of the security assessment we performed on the Rohs K88h smartwatch. In the next section, we are going to present another security assessment that we performed on a different device: the Sricam SP009 IP camera.

\section{Security of cheap and ubiquitous IoT devices: an IP camera case study}

With the spread of smart homes, our houses are increasingly populated of intelligent devices. As result, IP cameras, motion sensors, and connected door locks are more and more being used for home security [39].

An Internet Protocol camera, or simply IP camera, is a digital video camera commonly used for surveillance purposes, which may send and receive data via a computer network or the Internet [40]. It usually uses a Wi-Fi connection and implements a two-way audio communication which permits the user to listen and communicate with the environment in which the device is located. Moreover, it also makes possible to create a remote connection so that the customer can watch on a smartphone, or another device, the video being recorded.

There exist many low-cost IP cameras offering several functionalities and whose price is between $30 \$$ and $80 \$$. Most of these products typically store the images they acquire on a cloud server that can be accessed from everywhere.

IP cameras are composed by a lens, a sensor, a processor for analyzing images, and an internal memory where the firmware and software are installed. A typical functionality they offer is the possibility to act as alarm system. Indeed, the device can detect movements and react to them by sending a message to the registered e-mail addresses. Most of the time, an image is attached to the message so that the user can identify what caused the alarm and decide whether it is necessary to intervene or not. Further, most IP cameras are equipped with infrared sensors (IR sensors) which allow monitoring environments with low brightness. This set of features makes such devices suitable for being deployed in everyday environments.

Despite being used in everyday scenarios, the security of IP cameras is emerging as an important issue. An example of attack on surveillance cameras which occurred in a real scenario has been reported in [41].

In the next subsections, we analyze the security vulnerabilities of a low-cost IP camera. The device we considered is the Sricam SP009 H.264 Wifi Megapixel Wireless ONVIF CCTV IP camera, which was released by Shenzhen Sricctv Technology CO 7 . Before detailing our analysis, in the next subsection, we report some of the most relevant works related to the security of IP cameras.

\footnotetext{
7 http://www.sricam.com/
} 


\subsection{Related Works}

Although the literature on the topic is not very extended, for the sake of completeness, in this section, we review some works worth to be mentioned.

Albrecht and Mcintyre 42 provide examples of two IP cameras that have been hacked. The authors conclude the work proposing some palliative solutions, such as: to change default passwords, to set unique and strong passwords for all home devices, to update the firmware, and to set the devices not to broadcast their SSID.

Ali Tekeoglu and Ali Saman Tosun 40 investigate the security of cloud-based wireless IP cameras. They analyze the traffic generated by a low-end, easy-tosetup, off-the-shelf wireless IP camera for the average home user. The authors identify many security and privacy issues in these devices with different levels of severity. Specifically, they state that if a malicious person can sniff the IP camera network traffic anywhere in between the mobile device-cloud servers-IP camera path, he would be able to reconstruct the transmitted JPEG images, which is surely a serious privacy issue.

At the conference in Amsterdam on Positive Hacking Day, Sergey Shekyan and Artem Harutyunyan presented their analysis called "To Watch or Be Watched: Turning Your Surveillance camera Against You" 43. The presentation explains how to gain control over a camera in the wild, analyzes security malpractices and how to make the camera less insecure.

Reem Alshalawi and Turki Alghamdi [44] propose a solution against illegal access on wireless surveillance camera. The solution is designed into two stages. First, a new monitoring scheme is built to keep the privacy of data. Second, an investigation process is facilitated, playing an important role in saving users' privacy and highly secure places that use surveillance cameras.

The rest of this section focuses on security issues and vulnerabilities of the SP009 IP camera, concluding with possible solutions to mitigate the issues.

\subsection{System Overview}

IP cameras are key devices for video surveillance systems. Security and privacy standings of these devices are important and need to be studied in detail.

In our use case, we used the IP camera released by Sricam: the SP009 IP camera. Fig. 2 depicts this device and its main components: an IR Sensor, a Lens, a Microphone, a Speaker, a Reset, a MicroSD card slot, and a DC 5V Power Slot. Fig. 3 provides an overview of its main functionalities.

The camera presents the following technical requirements. It has to be connected to a router supporting the $2.4 \mathrm{GHz} \mathrm{Wi}-\mathrm{Fi}$ frequency band $(802.11 \mathrm{~b} / \mathrm{g} / \mathrm{n})$ with DHCP protocol enabled. Moreover, also the smartphone used to access the camera has to be connected to the Internet with a WLAN/Wi-Fi connection.

The device applies the advanced network transmission technology "Cloudlink P2P" which permits information exchange between the IP camera and a P2P Cloud. This $\mathrm{P} 2 \mathrm{P}$ architecture makes possible to watch a live feed from the camera. Indeed, using a smartphone, it is sufficient to download the appropriate 


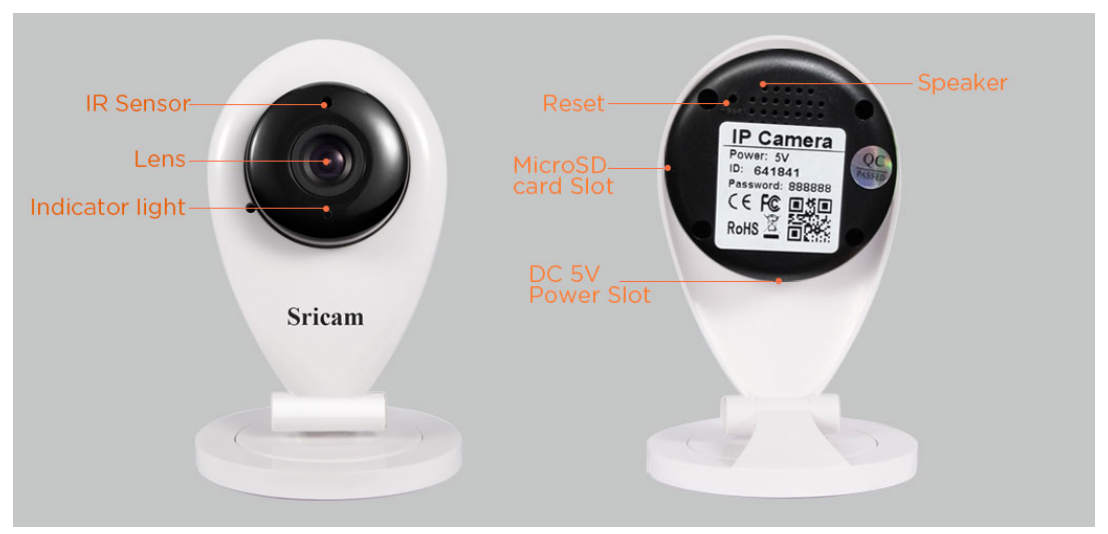

Fig. 2. The Sricam SP009 IP camera.

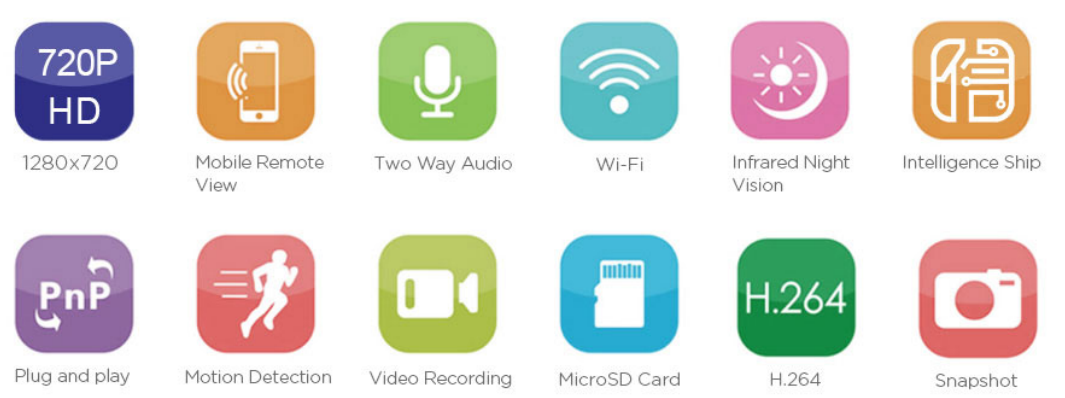

Fig. 3. Overview of functionalities offered by the Sricam SP009 IP camera.

application, scan the device QR code, and begin to watch a live feed from the camera. An overview of the systems is presented in Fig. 4.

\subsection{Tools and Set-Up}

In this section, the tools used to perform our security investigation are described.

The smartphone used for performing the experiments is a Huwawei P8 lite running Android 5.0.1. The mobile application necessary to control the IP camera is called "Sricam" and it is available both on Google Play ${ }^{8}$ and Apple Stor ${ }^{9}$. Moreover, for performing our experiments, we used the Sricam application having version 00.06.00.41. Sricam is a free mobile application customized for this brand of IP cameras. It allows to remotely monitor the camera, stream video, and organize settings of the camera. The application let also modify administration

8 https://play.google.com/store/apps/details?id=com.xapcamera\&hl=it

9 https://itunes.apple.com/it/app/sricam/id1040907995?mt=8 


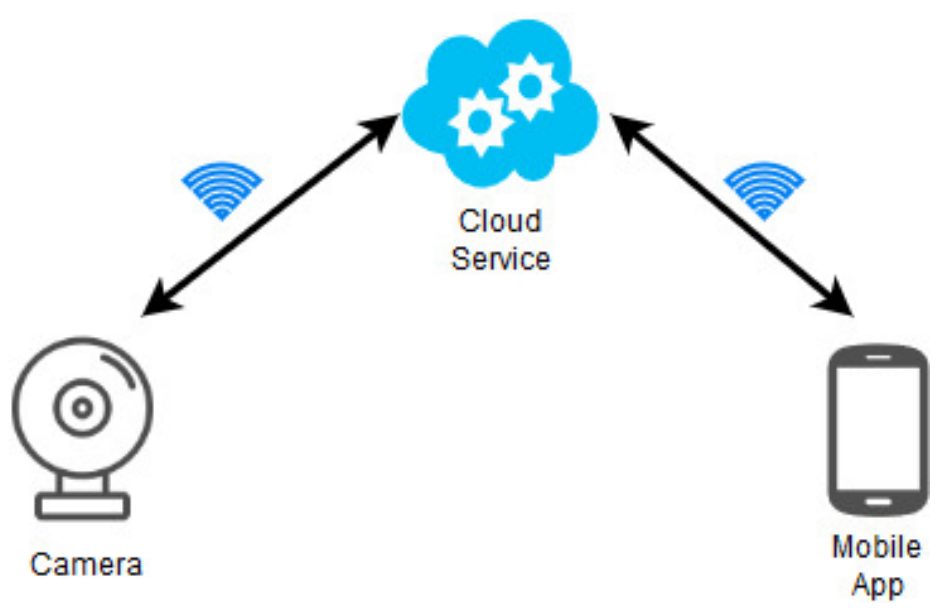

Fig. 4. System architecture of the Sricam SP009 IP camera.

credentials of the camera and manage a password for visitor users. Furthermore, customers can use the application to change network settings of the camera, such as: IP address, subnet, gateway, and DNS.

\subsection{The Vulnerabilities}

The purpose of this section is to describe the vulnerabilities we identified in the remote control system of the Sricam SP009 IP camera. Furthermore, we also present how such vulnerabilities can be exploited for compromising user's privacy.

Before presenting the vulnerabilities we identified, it is necessary to describe how the smartphone application used for controlling the camera works. Hence, in the next paragraphs, we are first of all going to describe how the application running on the smartphone works and which are the steps that users are required to follow for acquiring the rights to control a new camera. Subsequently, we describe the vulnerabilities we identified in this procedure and how a user can gain control of devices that he does not own.

After installing the application on the smartphone, it is required to register a new user account and to add the camera to the list of devices owned by the new user of the application. In order to perform this operation, it is required to specify the device ID and password, which are information reported on a label located beneath the camera support. The ID is a sequence number (in our case its value was: 995811), while, the password of each new camera has a default value of 888888 . After registering the device, the final step required for remotely controlling it is to connect the IP camera to a Wi-Fi network. At this point, it is possible to remotely access the camera, change its settings, set alarms and perform a lot of other operations enabled by the device. 
After this brief description of the procedure that allows gaining control of a new camera, we present the vulnerabilities we identified in this process.

Lack of adequate access control In the procedure for gaining control of a new camera, two main issues have been identified:

1. Although the application requires the user to register by means of a valid e-mail, we noticed that no confirmation e-mail is sent to the address used for registration. This implies the possibility to register a new account with a fake e-mail address or an address that is not owned by the user.

We strongly believe that sending a confirmation e-mail that forces the user to prove its identity is a minimal level of security that must be provided no matter what.

2. More important, once the new camera is added to the list of devices owned by the user, the application does not force him to change the default password of the device.

In relation to this, we have tested that, by entering a device ID similar to the ID of the device we own and by using the default password, it is possible to connect and control in a completely silent way other cameras that we do not possess. In Fig. 5, we depict the three simple steps required for gaining control of the image stream produced by a camera we do not own. Moreover, in the last image of Fig. 5 it is also possible to see a preview of the image stream we acquired.

If the first issue can be considered as having minor consequences, this is not true for the second one. We claim that if users are not compulsory forced to change the default password, it leads to the existence of a vast number of cameras that are used with a default password. This allows non-expert attackers to connect and gain control of such devices.

In order to have an insight on the number of IP cameras affected by this issue (i.e. the number of sold cameras that are still using the default password), we performed a simple experiment with the goal of estimating this number. Using the smartphone application, we randomly tested the devices with an ID number in the range from 995810 to 995840 . We discovered that, on this range of 30 cameras, 11 of them use the default password. In other words, in this small range, more than $30 \%$ of devices are affected by the vulnerability. If we assume that this percentage is representative for a larger set of samples, by knowing the number of sold cameras, we can try to empirically evaluate which is the number of cameras affected by the issue. According to the information reported on Amazon and eBay, we can conclude that at least 400 models of this specific camera have been sold. So, we can empirically derive that it could possible to control 146 cameras.

Even if these statistics might not be accurate, we believe that they demonstrate the potential impact this issue can have on the privacy of several families and businesses which use such devices. 


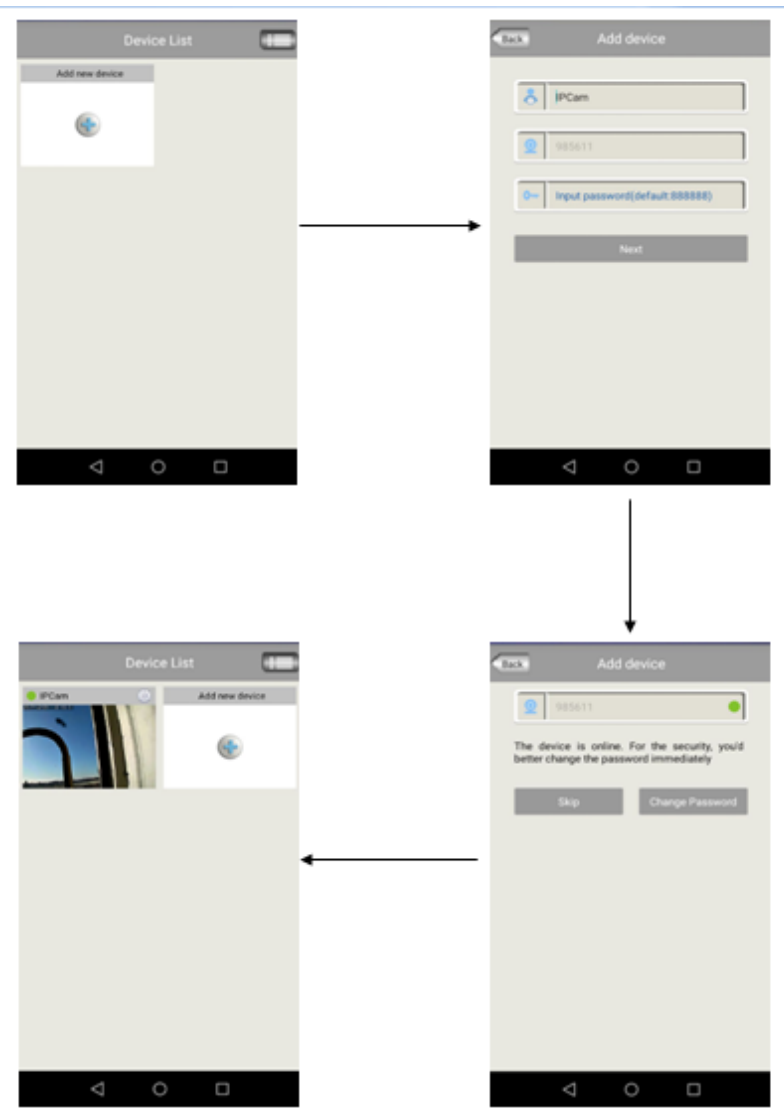

Fig. 5. Steps required to add a device that is not owned by the user of the application from which screenshots are taken. In the last image (located at the bottom, on the left), a preview of the scene captured by the camera we attacked is shown.

We argue that this vulnerability is critical for the privacy of users. Indeed, in order to understand this, it is sufficient to consider the multitude of actions attackers can perform without being real owners of the device:

- see and control the images being streamed by the camera: turn on/off the camera, use the local record, capture videos, and change the resolution of images;

- listen to what is happening in the environment in which the camera is placed by using the built-in microphone;

- affect the environment in which the camera is placed by using the built-in speaker (huge impact for baby-monitoring cameras);

- see and modify device information: device version, uBoot version, CPU version, and system version;

- see and modify time zone settings; 
- see and modify media settings: video standard (PAL/NTSC), volume, image reverse;

- modify security settings: add a visitor password;

- see and modify network settings: network mode (Wired or Wi-Fi), IP, subnet, gateway, DNS;

- see the name of Wi-Fi list and change Wi-Fi network;

- modify alarm settings: receive notifications (yes/no), e-mail alerts, motion detection alarm, buzzer alarm, alarm switch;

- see and modify email alerts information (sender, SMTP server, port, encryption mode) while being able to add a new email to which alarms have to be sent;

- see and modify record settings: modify record mode, see if the device uses an SD Card, record switch (yes/no);

- learn the version of device firmware.

The analysis we performed allowed to discover two important issues related to the Sricam SP009 IP camera. First of all, the smartphone application used for controlling the camera does not force users to change the default password after the camera has been registered in the system for the first time. Secondly, there is no proper access control, i.e. by only knowing the ID and the password of the camera, it is possible to have complete control of the device. Hence, by exploiting these two vulnerabilities, an external attacker can completely take control of the camera in an easy and silent fashion.

On top of that, in the next subsection, we describe another vulnerability that we identified by sniffing the network traffic generated by the smartphone application controlling the camera.

Insecure communication We decided to use Wireshark ${ }^{10}$ for inspecting the network packets exchanged between our application and the remote server. In doing this, we only focused on the packets exchanged during the process of adding a new camera to the list of devices. We discovered that the communication is not properly secured, since based on HTTP. Therefore, we were able to extract the ID of the device and its password by simply eavesdropping the communication channel. Hence, even if the password used by the IP camera is not the default one, it can still be possible to get such password by simply sniffing packets that are exchanged during the remote communication of the application.

The presence of all these vulnerabilities, triggered us to perform other experiments for verifying the existence of other issues. For this purpose, we performed the reverse engineering of the smartphone application controlling the IP camera.

Analysis and reverse engineering of the control application With the goal of verifying whether other vulnerabilities exist, we decided to analyze the smartphone application controlling the camera for better understanding how the overall control system is built and works.

\footnotetext{
$\overline{10}$ https://www.wireshark.org/download.html
} 
In order to perform the reverse engineering of the smartphone application, the following tools have been used:

- the application apk extractor ${ }^{11}$ has been employed for obtaining the installed apk from the smartphone;

- dex2jar ${ }^{12}$ has been used for converting the machine code to Java class files;

- Java compiler JD-GUI ${ }^{13}$ has been used for inspect the Java source code.

Using Android Studie ${ }^{14}$, we have partially analyzed the code of the Sricam application. We have been able to analyze which controls are performed when a user requests to add a new camera on his profile. We have discovered that the controls being performed for this purpose only involve the ID and password of the camera: the system checks if the IP camera details are correct, and successively, if the device is online, it is added in the device database of the user. Therefore, as suspected, we verified that no authentication or authorization of the user is involved in the process of adding a new device.

We are still working on the application source code for trying to infer more information on the way in which the overall system works in order to understand whether other vulnerabilities exist. In relation to this, we have already identified some possible issues that need to be analyzed in more detail.

\subsection{Possible Solutions}

Several possible solutions can be applied for patching the identified vulnerabilities. In the following, for each vulnerability we discovered, possible solutions are presented:

- Lack of adequate access control: this issue has to be addressed in three steps. First of all, the application has to be patched in order to force users to modify the device password after the IP camera has been successfully associated with a user for the first time.

Secondly, the access control mechanism must also take into account the user requesting to gain access to the device, i.e. it has not to rely solely on information of the device.

Finally, in order to implement proper access control mechanisms, it is paramount to have adequate authentication mechanisms. Hence, it is essential to solve the vulnerability associated with the improper verification of e-mail address at registration time.

- Insecure communication: using secure communication protocols would be sufficient to reduce the risk of eavesdropping sensitive information. In the specific case, we suggest using HTTPS instead of HTTP.

11 https://play.google.com/store/apps/details?id=com.ext.ui\&hl=it

12 https://sourceforge.net/projects/dex2jar/

13 http://jd.benow.ca/

14 https://developer.android.com/studio/index.html 


\section{Conclusions and future works}

The objective of this work is to raise the awareness in relation to the poor security state of cheap and ubiquitous IoT devices, with the aim of encouraging and supporting the development of new security solutions for IoT, such as the one presented in 45 .

It is our strong belief that this category of devices is a major threat to users' privacy. In order to demonstrate this, we selected two different devices and performed their security assessments. Only two criteria were used in selecting them: (1) they had to be among the cheapest in their market segments while offering several functionalities (2) they had to be ubiquitous (i.e. accompanying and supporting users in their daily activities). As a result, we inspected the security state of the Rohs K88h smartwatch and that of the Sricam SP009 IP camera. The outcomes of our analysis are clear: the security of such devices is neglected and, as a consequence, users' privacy is seriously threatened. Indeed, the results of both case studies show that even non-expert attackers can exploit such vulnerabilities for negatively affecting users' privacy.

We showed that the Rohs K88h smartwatch implements an insecure Bluetooth communication that allows the establishment of connections without requiring the approval of the end-user. In order to demonstrate how such vulnerability could be exploited by an adversary, we proposed to exploit the client application that interacts with the smartwatch for building an exploitation tool that silently exfiltrates sensitive information.

As regards the Sricam SP009 IP camera, we identified several vulnerabilities. The most important among these issues is the use of an inadequate access control mechanism. We showed that such simple issues can allow to completely take control of the IP camera together with the image stream it produces.

Our goal was to show the security vulnerabilities we identified in such devices. Nevertheless, we are currently working for building a complete data exfiltration tool for the Rohs K88h smartwatch that takes advantage of the reverse engineering of the existing application. Furthermore, in relation to the Sricam SP009 IP camera, we are studying the source code resulting from the reverse engineering of the application controlling the camera, for identifying the exact number of IP cameras that is possible to control. The overall objective of these future works is to demonstrate what is the concrete impact that these vulnerabilities have on privacy.

Based on our experience of assessing the security of IoT devices, future work will focus on how to secure the devices in context-aware systems. To this aim, we are currently working on a security framework based on two key concepts: Security-by-Contract 46,47 and Fog Computing 48 .

\section{References}

1. A. Zanella, N. Bui, A. Castellani, L. Vangelista, and M. Zorzi, "Internet of things for smart cities," IEEE Internet of Things journal, vol. 1, no. 1, pp. 22-32, 2014. 
2. M. Soliman, T. Abiodun, T. Hamouda, J. Zhou, and C.-H. Lung, "Smart home: Integrating internet of things with web services and cloud computing," in Cloud Computing Technology and Science (CloudCom), 2013 IEEE 5th International Conference on, vol. 2, pp. 317-320, IEEE, 2013.

3. S. D. T. Kelly, N. K. Suryadevara, and S. C. Mukhopadhyay, "Towards the implementation of iot for environmental condition monitoring in homes," IEEE Sensors Journal, vol. 13, no. 10, pp. 3846-3853, 2013.

4. L. Da Xu, W. He, and S. Li, "Internet of things in industries: A survey," IEEE Transactions on industrial informatics, vol. 10, no. 4, pp. 2233-2243, 2014.

5. A.-R. Sadeghi, C. Wachsmann, and M. Waidner, "Security and privacy challenges in industrial internet of things," in Proceedings of the 52nd annual design automation conference, p. 54, ACM, 2015.

6. R. Kirk, "Cars of the future: the internet of things in the automotive industry," Network Security, vol. 2015, no. 9, pp. 16 - 18, 2015.

7. P. A. Laplante and N. Laplante, "The internet of things in healthcare: Potential applications and challenges," IT Professional, vol. 18, pp. 2-4, May 2016.

8. S. Amendola, R. Lodato, S. Manzari, C. Occhiuzzi, and G. Marrocco, "Rfid technology for iot-based personal healthcare in smart spaces," IEEE Internet of things journal, vol. 1, no. 2, pp. 144-152, 2014.

9. N. Eddy, "Gartner: 21 billion iot devices to invade by 2020," Information Week, Nov, vol. 10, 2015.

10. S. Sicari, A. Rizzardi, L. A. Grieco, and A. Coen-Porisini, "Security, privacy and trust in internet of things: The road ahead," Computer networks, vol. 76, pp. 146$164,2015$.

11. Q. Jing, A. V. Vasilakos, J. Wan, J. Lu, and D. Qiu, "Security of the internet of things: perspectives and challenges," Wireless Networks, vol. 20, no. 8, pp. 24812501, 2014.

12. N. Dragoni, A. Giaretta, and M. Mazzara, "The internet of hackable things," in International Conference in Software Engineering for Defence Applications, pp. 129140, Springer, 2016.

13. R. Goyal, N. Dragoni, and A. Spognardi, "Mind the Tracker You Wear: A Security Analysis of Wearable Health Trackers," in Proceedings of the 31st Annual ACM Symposium on Applied Computing, SAC '16, (New York, NY, USA), pp. 131-136, ACM, 2016.

14. A. Giaretta, M. De Donno, and N. Dragoni, "Adding Salt to Pepper: A Structured Security Assessment over a Humanoid Robot," in Proceedings of the 13th International Conference on Availability, Reliability and Security, ARES 2018, (New York, NY, USA), pp. 22:1-22:8, ACM, 2018.

15. R. Rawassizadeh, B. A. Price, and M. Petre, "Wearables: Has the age of smartwatches finally arrived?," Communications of the $A C M$, vol. 58, no. 1, pp. 45-47, 2015.

16. B. Mortazavi, E. Nemati, K. VanderWall, H. G. Flores-Rodriguez, J. Y. J. Cai, J. Lucier, A. Naeim, and M. Sarrafzadeh, "Can smartwatches replace smartphones for posture tracking?," Sensors, vol. 15, no. 10, pp. 26783-26800, 2015.

17. R. Wu, E. De Lara, D. Liaqat, I. Thukral, and A. S. Gershon, "Feasibility of using smartwatches and smartphones to monitor patients with copd," in A49. COPD: CARE DELIVERY, pp. A1695-A1695, American Thoracic Society, 2016.

18. G. M. Weiss, J. L. Timko, C. M. Gallagher, K. Yoneda, and A. J. Schreiber, "Smartwatch-based activity recognition: A machine learning approach," in Biomedical and Health Informatics (BHI), 2016 IEEE-EMBS International Conference on, pp. 426-429, IEEE, 2016. 
19. F. B. A. Ramos, A. Lorayne, A. A. M. Costa, R. R. de Sousa, H. O. Almeida, and A. Perkusich, "Combining smartphone and smartwatch sensor data in activity recognition approaches: an experimental evaluation.," in SEKE, pp. 267-272, 2016.

20. E. Casilari and M. A. Oviedo-Jiménez, "Automatic fall detection system based on the combined use of a smartphone and a smartwatch," PloS one, vol. 10, no. 11, p. e0140929, 2015.

21. A. H. Johnston and G. M. Weiss, "Smartwatch-based biometric gait recognition," in Biometrics Theory, Applications and Systems (BTAS), 2015 IEEE 7th International Conference on, pp. 1-6, IEEE, 2015.

22. W.-H. Lee and R. Lee, "Implicit sensor-based authentication of smartphone users with smartwatch," in Proceedings of the Hardware and Architectural Support for Security and Privacy 2016, p. 9, ACM, 2016.

23. D. Guerra-Rodríguez and A. Granollers, "User experience experiments with mobile devices in outdoors activities: Use case: Cycling and mountain biking," in Proceedings of the XVII International Conference on Human Computer Interaction, Interaccion '16, (New York, NY, USA), pp. 26:1-26:4, ACM, 2016.

24. X. Liu, Z. Zhou, W. Diao, Z. Li, and K. Zhang, "When good becomes evil: Keystroke inference with smartwatch," in Proceedings of the 22Nd ACM SIGSAC Conference on Computer and Communications Security, CCS '15, (New York, NY, USA), pp. 1273-1285, ACM, 2015.

25. A. Sarkisyan, R. Debbiny, and A. Nahapetian, "Wristsnoop: Smartphone pins prediction using smartwatch motion sensors," in 2015 IEEE International Workshop on Information Forensics and Security (WIFS), pp. 1-6, Nov 2015.

26. H. Wang, T. T.-T. Lai, and R. Roy Choudhury, "Mole: Motion leaks through smartwatch sensors," in Proceedings of the 21st Annual International Conference on Mobile Computing and Networking, MobiCom '15, (New York, NY, USA), pp. 155166, ACM, 2015.

27. A. Maiti, M. Jadliwala, J. He, and I. Bilogrevic, "(smart)watch your taps: Sidechannel keystroke inference attacks using smartwatches," in Proceedings of the 2015 ACM International Symposium on Wearable Computers, ISWC '15, (New York, NY, USA), pp. 27-30, ACM, 2015.

28. A. Maiti, M. Jadliwala, J. He, and I. Bilogrevic, "Side-channel inference attacks on mobile keypads using smartwatches," IEEE Transactions on Mobile Computing, vol. PP, no. 99, pp. 1-1, 2018.

29. C. X. Lu, B. Du, H. Wen, S. Wang, A. Markham, I. Martinovic, Y. Shen, and N. Trigoni, "Snoopy: Sniffing your smartwatch passwords via deep sequence learning," Proc. ACM Interact. Mob. Wearable Ubiquitous Technol., vol. 1, pp. 152:1152:29, Jan. 2018.

30. Q. Do, B. Martini, and K. R. Choo, "Is the data on your wearable device secure? an android wear smartwatch case study," Software: Practice and Experience, vol. 47, no. 3, pp. 391-403.

31. J. Liu and W. Sun, "Smart attacks against intelligent wearables in people-centric internet of things," IEEE Communications Magazine, vol. 54, pp. 44-49, December 2016.

32. S. Siboni, A. Shabtai, N. O. Tippenhauer, J. Lee, and Y. Elovici, "Advanced security testbed framework for wearable iot devices," ACM Trans. Internet Technol., vol. 16, pp. 26:1-26:25, Dec. 2016.

33. Y. Lee, W. Yang, and T. Kwon, "Poster: Watch out your smart watch when paired," in Proceedings of the 2017 ACM SIGSAC Conference on Computer and Communications Security, CCS '17, (New York, NY, USA), pp. 2527-2529, ACM, 2017. 
34. "Internet of things security study: Smartwatches." https://www.ftc.gov/system/ files/documents/public_comments/2015/10/00050-98093.pdf Accessed: 2018$03-26$.

35. J. Padgette, "Guide to bluetooth security," NIST Special Publication, vol. 800, p. 121, 2017.

36. Estefanía Martínez, "Best chinese smartwatch of 2017." epinium.com, 2017. http: //epinium.com/blog/best-chinese-smartwatch/, accessed Oct 2017.

37. Amazon.co.uk, "K88h product description." Amazon.co.uk, 2017. https: //www.amazon.co.uk/Waterproof-Dustproof-Notification-SmartphoneSilver-Black-Steel/dp/B01MSYUOFM/ref=sr_1_1?s=electronics\&ie=UTF8\&qid= 1507642684\&sr=1-1\&keywords=k88h, accessed Oct 2017.

38. S. Bluetooth, "Rfcomm with ts 07.10," Bluetooth SIG, 2003.

39. E. Fernandes, J. Jung, and A. Prakash, "Security analysis of emerging smart home applications," in 2016 IEEE Symposium on Security and Privacy (SP), pp. 636654, May 2016.

40. A. Tekeoglu and A. S. Tosun, "Investigating security and privacy of a cloud-based wireless ip camera: Netcam," in 2015 24th International Conference on Computer Communication and Networks (ICCCN), pp. 1-6, IEEE, 2015.

41. "Cctv hack takes casino for $\$ 33$ million in poker losses." https:// www.theregister.co.uk/2013/03/15/cctv_hack_casino_poker/ Accessed: 201803-30.

42. K. Albrecht and L. Mcintyre, "Privacy nightmare: When baby monitors go bad [opinion]," IEEE Technology and Society Magazine, vol. 34, no. 3, pp. 14-19, 2015.

43. S. Shekyan and A. Harutyunyan, "To watch or be watched: Turning your surveillance camera against you."

44. R. Alshalawi and T. Alghamdi, "Forensic tool for wireless surveillance camera," in 2017 19th International Conference on Advanced Communication Technology (ICACT), pp. 536-540, Feb 2017.

45. M. De Donno, N. Dragoni, A. Giaretta, and M. Mazzara, "AntibIoTic: protecting IoT devices against DDoS attacks," in International Conference in Software Engineering for Defence Applications, pp. 59-72, Springer, 2016.

46. N. Dragoni, F. Massacci, T. Walter, and C. Schaefer, "What the Heck is this Application Doing? A Security-by-Contract Architecture for Pervasive Services," Computers \& Security, vol. 28, no. 7, pp. 566-577, 2009.

47. N. Bielova, N. Dragoni, F. Massacci, K. Naliuka, and I. Siahaan, "Matching in Security-By-Contract for Mobile Code," Journal of Logic and Algebraic Programming, vol. 78, pp. 340-358, 2009.

48. F. Bonomi, R. Milito, J. Zhu, and S. Addepalli, "Fog Computing and Its Role in the Internet of Things," in Proceedings of the First Edition of the MCC Workshop on Mobile Cloud Computing, MCC '12, (New York, NY, USA), pp. 13-16, ACM, 2012 . 